Associate professor Shouheng TUO, PhD

School of Computer Science and Technology, Xi'an University of Posts and Telecommunications, Xi'an Shaanxi, China

E-mail: tuo_sh@126.com

Associate professor Hong HE

College of Economics and Management, Xi'an University of Posts \& Telecommunications, Xi'an Shaanxi, China

E-mail: hehongtbw@126.com

\title{
DEAf-MOPS/D: AN IMPROVED DIFFERENTIAL EVOLUTION ALGORITHM FOR SOLVING COMPLEX MULTI-OBJECTIVE PORTFOLIO SELECTION PROBLEMS BASED ON DECOMPOSITION
}

\begin{abstract}
With the high-speed economic development and economic diversification of the world, it is very necessary to develop an effective and efficient portfolio selection method with high precision and robustness. In this study, we first introduce an enhanced multi-objective cardinality constrained mean-variance (CCMV) model, in which the transaction costs and price-earning (P/E) ratio are appended in the model, then an improved differential evolution algorithm with adaptive fine-tune is proposed to solve multi-objective portfolio selection problems based on decomposition (DEaf-MOPS/D).Finally, five simulation experiments on five benchmark datasets (HangSeng, DAX 100, FTSE 100, S\&P 100, and Nikkei 225) are employed to investigate the performance of our method. The experimental results indicate that the performance of DEaf-MOPS/D is superior to other compared algorithms, and its runtime is much less than other algorithms, which demonstrate that our method is efficient in solving high-dimension portfolio selection problems.
\end{abstract}

Keywords: Portfolio Selection problems, Multi-objective, Differential Evolution, price-earning.

JEL Classification: G11 
Shouheng Tuo, Hong He

\section{Introduction}

In 1981, Nobel Prize winner James Tobin said: "Do not put eggs in a basket.", which means reducing risk by diversifying investments. In wealth management, we aim to maximum return and reduce the risk by following the principle of diversifying investments. Therefore, choosing the best assets and the right amount of assets has become a very important research topic, which is a NP-hard problem. To tackle this problem, some mathematical models and solving methods have been proposed. Mean-variance (MV) model that is the first mathematical model was presented by Markowitz in 1959 (Markowitz, H. M. 1959), it measures the return and risk by mean and variance of portfolio returns. The MV established a touchstone in the portfolio theory. Next, some alternative risk measures are proposed for estimating the risk, such as Value-at-Risk (VaR) (P. Jorion, 1997), Mean-Absolute Deviation (MAD) (Yamazaki, K. H. 1991) and Conditional VaR (R.T. Rockafellar and S. Uryasev, 2000). However, it is estimated that little is known. "Do not put the eggs in a basket". In fact, there is a second sentence, that is - "but don't put it in too many baskets". Hence, some constraints must be considered by real-life investors, such as cardinality constraints (CC) used to restrict the number of assets in the portfolio (M.J. Magill and G.M. Constantinides, 1976), transaction costs (TC) (K. Metaxiotis, K and Liagkouras, 2012) for reducing the transaction costs from the outcome (Ertenlice $\mathrm{O}$ and Kalayci C B, 2018).

In this work, we proposed a swarm intelligent optimization algorithm (DEaf-MOPS/D) to tackle high-dimensional portfolio selection problems. In section II, we introduce cardinality constraint mean-variance (CCMV) model and merge the transaction costs and price-earnings ratio into the CCMV model. Section III analyses the challenges of solving the CCMV models. Related works of solving the MV and CCMV models are introduced in section IV. Our proposed algorithm is discussed in detail in section VI. Section VII and VIII are respectively the simulation experiments and conclusion.

\section{Mathematical Model}

The standard MV models have two objectives, the first one is for maximum investment return of selected assets, the second one aims at minimum the risk of investment. In realistic investments, the bound constraints, CC, TC and 
DEaf-MOPS/D: An Improved Differential Evolution Algorithm for Solving Complex Multi-objective Portfolio Selection Problems Based on Decomposition

price-earnings ratio $(\mathrm{P} / \mathrm{E})$ of asset all are very important factors to be considered by the investors. In this study, we employ multiple constraints MV model as follows,

$$
\begin{aligned}
& \text { Minimize } \mathrm{F}(\mathbf{x})=\left(f_{1}(\mathbf{x}), f_{2}(\mathbf{x}), f_{3}(\mathbf{x})\right) \\
& \left\{\begin{array}{l}
f_{1}(\mathbf{x})=\min _{\mathbf{x}}\left(\varphi(\mathbf{x})-\mathbf{x}^{\top} \mathbf{\mu}\right)=\min \sum_{i=1}^{n}\left(\varphi\left(x_{i}\right)-x_{i} \mu_{i}\right) \\
f_{2}(\mathbf{x})=\min _{\mathbf{x}}\left(\mathbf{x}^{\top} \Sigma \mathbf{x}\right)=\min \sum_{i=1}^{n} \sum_{j=1}^{n} x_{i} \sigma_{i j} x_{j} \\
f_{3}(\mathbf{x})=\min _{\mathbf{x}}\left(\frac{\boldsymbol{P}}{\boldsymbol{E}}\right)^{\mathbf{T}} \cdot \mathbf{x}=\min \sum_{i=1}^{n} \frac{p_{i} x_{i}}{e_{i}}
\end{array}\right.
\end{aligned}
$$

\section{Subject to}

$$
\left\{\begin{array}{l}
\sum_{i=1}^{n} x_{i}=1 \\
\varepsilon^{L} z_{i} \leq x_{i} \leq \varepsilon^{U} z_{i}\left(z_{i} \in\{0,1\}\right) \\
\sum_{i=1}^{n} z_{i}=K
\end{array}\right.
$$

where the objective function $f_{1}(\mathbf{x})$ aims to maximum the expected returns. $f_{2}(\mathbf{x})$ represents the investment risk. $f_{3}(\mathbf{x})$ is used to choose the assets with minimum the price-earnings $(\mathrm{P} / \mathrm{E})$ ratio. $\mathbf{x}=\left(x_{1}, x_{2}, \ldots, x_{\mathrm{n}}\right)$ denotes the vector of portfolio weights, $0<x_{i}<1(i=1,2, \ldots, \mathrm{n}) . \boldsymbol{\mu}=\left(\mu_{1}, \mu_{2}, \ldots, \mu_{\mathrm{n}}\right)$ represents the vector of expected return rate of all assets. $\varphi\left(x_{\mathrm{i}}\right)$ is the transaction cost of $\mathrm{i}^{\text {th }}$ asset. $\Sigma_{\text {is }}$ the covariance matrix of rates of asset returns, $\sigma_{i j}$ denotes the return covariance of assets $i$ and $j . p_{\mathrm{i}}$ and $e_{\mathrm{i}}$ represent the market value and earnings of $\mathrm{i}^{\text {th }}$ asset. $\varepsilon^{L}$ and $\varepsilon^{U}$ are respectively the lower bound and upper bound of $x_{\mathbf{i}} . K$ is the number of assets expected to be invested. $z_{i}=1$ if the $\mathrm{i}^{\text {th }}$ asset are chosen, otherwise $z_{i}=0$.

In the mathematical model, we append the price-earnings (P/E) ratio to the cardinality constraint mean variance (CCMV) model because, in a real portfolio, 
Shouheng Tuo, Hong He

the P/E is also a very important factor in experts' considerations. The P/E denotes profit gained through the stock relative to its market price that can be important and effective for investments.

\section{Challenges for solving the high-dimensional portfolio selection problems}

The CCMV is a very complex NP-hard problem, solving it faces the follow challenges.

(1) Combinatorial Explosion. Portfolio selection is a high-dimensional combination optimization problem, heavy computation burden is the first challenge.

(2) Multi-objective optimization. The CCMV model has multiple objective functions that are mutually exclusive, for example, the expected returns and the risk are contradictory. How to balance the multi-objective functions is the second challenge.

(3) Constraint Handling. There are multiple constraint conditions in the CCMV models, such as the number $(K)$ of assets expected to be invested, portfolio weight $\varepsilon^{L} \leq x_{i} \leq \varepsilon^{U}$. These constraints are usually contradictory with the objective function. Therefore, constraint handling is the third challenge.

\section{Related work}

Swarm intelligent optimization algorithm, which is not limited to the characteristics of mathematical models, has attracted considerable attention in solving high-dimensional portfolio selection problem. Chang et al. (T.-J. Chang et al. 2008) and M.Woodside-Oriakhi et al.( M. Woodside-Oriakhi et al.,2011) employed genetic algorithm (GA) to solve the CCMV model, in genetic algorithm, a candidate solution of portfolio selection was represented by a binary vector. K.Liagkouras and K.Metaxiotis proposed a multio-bjective evolutionary algorithm to solve multi-period mean-variance fuzzy portfolio optimization model (K.Liagkouras and K.Metaxiotis, 2018). In literature, particle swarm optimization (PSO) are used to solving constrained portfolio optimization problems $(\mathrm{H}$. Zhu, 2011). S. Kamali proposed a hybrid algorithm that merges PSO and GA to 
DEaf-MOPS/D: An Improved Differential Evolution Algorithm for Solving Complex Multi-objective Portfolio Selection Problems Based on Decomposition optimize portfolio (S. Kamali, 2014). B. Niu et al. and L.Tan et al. adopted bacterial foraging optimization to solve constrained portfolio optimization problems (B. Niu et al., 2012, L.Tan, et al. 2013). Invasive weed optimization is employed for solving multi-objective portfolio optimization problem (A.R. Pouya, et al., 2016). I Strumberger introduced a hybrid Bat Algorithm for constrained portfolio optimization (Strumberger I, et al., 2017). C.B. Kalayci presented an artificial bee colony algorithm with feasibility enforcement and infeasibility toleration procedures for CCMV portfolio optimization (C.B. Kalayci, et al., 2017). In addition, harmony search and teaching-learning-based optimization were employed to solve high-dimensional portfolio selection problems (Tuo $\mathrm{SH}$ and $\mathrm{He}$ H, 2016, 2018). These studies have been good progress in solving portfolio selection problems. However, most of algorithms take a long time to obtain the optimal frontier because many various weight coefficients of objective functions are considered. In this study, we propose a fast optimization algorithm, named DEaf-MOPS/D, which improves differential evolution algorithm using adaptive fine-tuning strategy to solve multi-objective portfolio selection problems based on decomposition.

\section{Proposed algorithm DEaf-MOPS/D}

The proposed DEaf-MOPS/D algorithm employs the idea of MOEA/D (Zhang Q and Hui L, 2007), it decomposes multi-objective portfolio selection problem into a number of scalar optimizations subproblems and then each subproblem is optimized by employing differential evolution (DE) according to the information from its neighboring subproblems. The DEaf-MOPS/D can obtain all the dominated solutions simultaneously, it has much lower computational complexity than other kind of optimization algorithms, such as NSGA-II (K. Deb, et al., 2002) and SPEA-II (E. Zitzler, et al., 2001). The steps of DEaf-MOPS/D are shown in Figure 1.

Algorithm 1. DEaf-MOPS/D algorithm

Step 1. Parameter initialization. 
Shouheng Tuo, Hong He

$\mathrm{D}$ - Dimension of solution, it is equal to the total number of assets investor can select. $\mathrm{m}$ - The number of objective functions.

$\varepsilon^{L}, \varepsilon^{U}-$ the lower and upper bound of portfolio weights.

$\mathrm{K}$ - The number of assets expected to be invested.

$\mathrm{T}-\mathrm{-}$ The number of weight vectors in the neighborhood of each weight vector.

(1) Generate $\mathrm{H}$ weight coefficients $\left(\omega_{1}, \omega_{2}, \cdots, \omega_{\mathrm{H}}\right)$ uniformly in [0,1], and then construct $N$ weight vector $\left(\lambda^{1}, \lambda^{2}, \cdots, \lambda^{N}\right)\left(\mathrm{N}=\mathrm{C}_{\mathrm{H}+\mathrm{m}-1}^{\mathrm{m}-1}\right)$ where $\lambda^{i}=\left(\omega_{1}, \omega_{2}, \cdots, \omega_{\mathrm{m}}\right), \sum_{j=1}^{m} \omega_{j}=1$.

(2) Normalize weight vector. $\lambda^{i}=\lambda^{i} / \sum_{i=1}^{N} \lambda^{i}$.

Step 2. Assign the neighborhoods for each weight vector.

Find the $\mathrm{T}$ closest weight vectors $\left(\lambda_{1}^{i}, \lambda_{2}^{i}, \cdots, \lambda_{T}^{i}\right)$ to $\mathrm{i}^{\text {th }}$ weight vector $\lambda^{i}$ according to the distance from $\lambda^{i}$ to other weights vectors.

\section{Step 3. Population initialization.}

(1) Generate population $\left(\mathbf{x}^{1}, \mathbf{x}^{2}, \cdots, \mathbf{x}^{\mathbf{N}}\right)$ uniformly randomly in the feasible space.

(2) Initialize the reference point by setting $\mathrm{ZZ}_{*}^{\mathrm{k}}=\min \left\{\mathrm{f}_{\mathrm{k}}\left(\mathrm{x}^{\mathrm{i}}\right), \mathrm{f}_{\mathrm{k}}\left(\mathrm{x}^{\mathrm{i}}\right), \cdots, \mathrm{f}_{\mathrm{k}}\left(\mathrm{x}^{\mathrm{i}}\right)\right\}, \mathrm{k}=1,2, \ldots, \mathrm{T} ; \mathrm{i}=1,2, \ldots, \mathrm{N}$.

(3) Execute constraint handles as literature [18-19].

(4) Evaluate each individual using objective functions.

$$
f z^{i}=\left(f_{1}\left(\mathbf{x}^{\mathbf{i}}\right), f_{2}\left(\mathbf{x}^{\mathbf{i}}\right), \cdots, f_{\mathrm{T}}\left(\mathbf{x}^{\mathbf{i}}\right)\right)
$$

(5) gen $=1$

Step 3. Differential evolution for each individual $\mathbf{x}^{\mathbf{i}}(i=1,2, \ldots, N)$.

(1) Mutation operation (see Algorithm 2).

(2) Crossover operation (see Algorithm 2).

(3) Selection operation (see Algorithm 2).

Step 4. gen $=$ gen +1 .

If gen $<$ Gen

go to Step 3.

Figure 1. Steps of DEaf-MOPS/D algorithm

Algorithm 2. Differential evolution for individual $x$ 
DEaf-MOPS/D: An Improved Differential Evolution Algorithm for Solving Complex Multi-objective Portfolio Selection Problems Based on Decomposition

(1) Get the T closest weight vectors $\left(\lambda^{i, 1}, \lambda^{i, 2}, \cdots, \lambda^{i, T}\right)$ to $\mathrm{i}^{\text {th }}$ weight vector $\lambda^{i}$.

(2) Select three individuals $\left(\mathbf{x}^{\mathbf{r} 1}, \mathbf{x}^{\mathbf{r} 2}, \mathbf{x}^{\mathbf{r}}\right)$ from population randomly.

(3) Mutation operation. $\mathbf{x}_{\text {new }}^{\mathrm{i}}=\mathbf{x}^{\mathrm{r} 1}+\mathbf{F}\left(\mathbf{x}^{\mathbf{r}^{2}}-\mathbf{x}^{\mathbf{r} 3}\right)$, F is the scale factor.

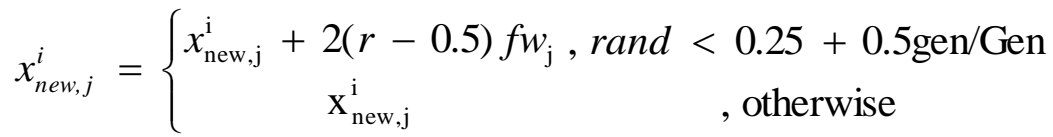

$x_{n e w, j}^{i}$ denotes the $\mathrm{j}^{\text {th }}$ dimension of $\mathbf{x}_{\text {new }}^{i}, r$ and rand represent the random number between 0 and 1 . Gen and gen denote the maximum number of iterations and the current number of iterations, respectively. $f w$ is the fine-tuning step, it is changed as follow,

$$
f w_{j}=\max \left(10^{-6}, \frac{\varepsilon^{\mathrm{U}}-\varepsilon^{\mathrm{L}}}{100 \cdot \text { gen }}\right)
$$

(4) Crossover operation. $x_{n e w, j}^{i}=\left\{\begin{array}{l}x_{\text {new, } j}^{i}, \text { rand }<c p \\ x_{\text {old }, j}^{i}, \text { otherwise }\end{array}\right.$

(5) Execute constraint handles as literature (T.Cura et al., 2009 ).

(6) Evaluate $\mathbf{x}_{\text {new }}^{i} . \quad f z_{\text {new }}^{i}=\left(f_{1}\left(\mathbf{x}_{\text {new }}^{i}\right), f_{2}\left(\mathbf{x}_{\text {new }}^{i}\right), \cdots, f_{\mathrm{T}}\left(\mathbf{x}_{\text {new }}^{i}\right)\right)$

(7) Selection operation.

$$
\begin{aligned}
& \mathrm{d}_{\text {new }}=\max \lambda^{\mathrm{i}}\left|\mathrm{fz}_{\text {new }}^{\mathrm{i}}-\mathrm{zz}\right| \\
& =\max \left\{\lambda_{1}^{\mathrm{i}}\left|\mathrm{fz}_{\text {new }, 1}^{\mathrm{i}}-\mathrm{zz}_{1}\right|, \lambda_{2}^{\mathrm{i}}\left|\mathrm{fz}_{\text {new }, 2}^{\mathrm{i}}-\mathrm{zz}_{2}\right|, \cdots, \lambda_{\mathrm{T}}^{\mathrm{i}}\left|\mathrm{fz}_{\text {new }, \mathrm{T}}^{\mathrm{i}}-\mathrm{zz}_{\mathrm{T}}\right|\right\} \\
& \text { If } \mathrm{d}_{\text {new }}<\mathrm{d}_{\text {old }} \text {, update } \mathbf{x}^{i}=\mathbf{x}_{\text {new }}^{i}, \mathrm{fz}^{\mathrm{i}}=\mathrm{fz}_{\text {new }}^{\mathrm{i}}
\end{aligned}
$$

Figure 2. The process of improved differential evolution strategy

\section{Experiments}

The proposed algorithm DEaf-MOPS/D has been investigated on five benchmark datasets (HangSeng, DAX100, FTSE100, S\&P100 and Nikkei) that can be download from OR-Library (http://people.brunel.ac.uk/ mastjjb/jeb/info.html). We use DEaf-MOPS/D to conduct the experiments and analysis for unconstraint, cardinality constraint, transaction cost constraint, and the experimental results are compared with four swarm intelligent algorithms: HS-TLBO (Tuo SH, 2016), HSDS (Tuo SH and He H, 2018), GA (Chang TJ et al., 2000), PSO (T.Cura, 2009). 
Shouheng Tuo, Hong He

The parameter settings of DEaf-MOPS/D are as follows:

Scale factor $\mathrm{F}=0.5$; Crossover probability $\mathrm{cp}=0.35 ; \mathrm{T}=10$.

Gen $=\frac{2000 m D}{N P}(\mathrm{~m}$ is the number of objective functions, D denotes the number of assets that can be selected. NP is the size of population of DEaf-MOPS/D).

Performance indexes: in the experiment, mean Euclidian distance (MED), variance of returns error (VRE), mean return error (MRE) ((T.Cura et al., 2009 )) and runtime are adopted to evaluate the performance of algorithms.

\subsection{Unconstraint tests.}

All of the constraint conditions are not considered.

(Test 1.1) Two objective functions. The objective function of $\mathrm{P} / \mathrm{E}$ is not employed in the test. The experimental results are summarized in Table 1.

(Test 1.2) Three objective functions. All three objective functions (maximum investment return, minimum risk, minimum P/E) are considered. In this test, our method is investigated. The optimal frontiers are shown in Figure 3 and the results (MED, VRE, MRE and runtime) are presented in Table 2.

As showed in Table 1, on metrics MED, VAR and MRE, the HS-TLBO algorithm is superior to other four algorithms except for dataset HangSeng. However, the runtime of DEaf-MOPS/D is much less than other four algorithms on all datasets, it is about a tenth of runtime of HS-TLBO. The performance of DEaf-MOPS/D on other metrics is also competitive compare to HS-TLBO for the unconstraint portfolio selection problems.

It can be observed in Figure 3 that optimal frontiers obtained by using DEaf-MOPS/D algorithm on five datasets are clearly hierarchical and evenly distributed. The larger the P/E value is, the greater the risk and the return are. In Table 2, the MED, VRE and MRE are calculated according to the risks and returns of optimal frontiers, which also displays very high-precision values on MED and MRE.

Table 1. The experimental results of five algorithms on five datasets

\begin{tabular}{lllllll}
\hline Data & $\begin{array}{l}\text { Metric } \\
\text { index }\end{array}$ & DEaf-MOPS/D & HS-TLBO & HSDS & GA & PSO \\
\hline
\end{tabular}


DEaf-MOPS/D: An Improved Differential Evolution Algorithm for Solving Complex Multi-objective Portfolio Selection Problems Based on Decomposition

\begin{tabular}{lllllll}
\hline & MED & $2.91 \mathrm{E}-06$ & $\mathbf{7 . 7 5 E}-07$ & $6.59 \mathrm{E}-07$ & $5.90 \mathrm{E}-04$ & $7.40 \mathrm{E}-04$ \\
HangSeng & VRE & $1.59 \mathrm{E}-01$ & $1.94 \mathrm{E}-02$ & $1.60 \mathrm{E}-02$ & $2.90 \mathrm{E}-01$ & $3.90 \mathrm{E}-01$ \\
& MRE & $1.77 \mathrm{E}-02$ & $8.89 \mathrm{E}-03$ & $7.89 \mathrm{E}-03$ & $1.10 \mathrm{E}-01$ & $1.30 \mathrm{E}-01$ \\
& Runtime(s) & $\mathbf{1 . 0 7 E}+01$ & $1.47 \mathrm{E}+02$ & $1.81 \mathrm{E}+02$ & $1.08 \mathrm{E}+02$ & $7.51 \mathrm{E}+01$ \\
\hline \multirow{5}{*}{ DAX 100 } & MED & $2.79 \mathrm{E}-06$ & $\mathbf{1 . 8 0 E}-06$ & $1.47 \mathrm{E}-04$ & $1.20 \mathrm{E}-03$ & $1.40 \mathrm{E}-03$ \\
& VRE & $4.75 \mathrm{E}-01$ & $9.60 \mathrm{E}-02$ & $7.09 \mathrm{E}+00$ & $3.10 \mathrm{E}-01$ & $3.90 \mathrm{E}-01$ \\
& MRE & $1.28 \mathrm{E}-02$ & $9.96 \mathrm{E}-03$ & $1.26 \mathrm{E}+00$ & $1.20 \mathrm{E}-01$ & $1.30 \mathrm{E}-01$ \\
& Runtime(s) & $\mathbf{8 . 7 3 E}+01$ & $1.27 \mathrm{E}+03$ & $1.57 \mathrm{E}+03$ & $9.30 \mathrm{E}+02$ & $6.50 \mathrm{E}+02$ \\
\hline \multirow{5}{*}{ FTSE 100 } & MED & $2.20 \mathrm{E}-06$ & $\mathbf{4 . 7 5 E - 0 7}$ & $3.72 \mathrm{E}-05$ & $3.00 \mathrm{E}-04$ & $3.30 \mathrm{E}-04$ \\
& VRE & $4.82 \mathrm{E}-01$ & $2.37 \mathrm{E}-02$ & $2.66 \mathrm{E}+00$ & $5.00 \mathrm{E}-01$ & $5.40 \mathrm{E}-01$ \\
& MRE & $9.50 \mathrm{E}-03$ & $5.86 \mathrm{E}-03$ & $3.09 \mathrm{E}-01$ & $5.70 \mathrm{E}-02$ & $6.40 \mathrm{E}-02$ \\
& Runtime(s) & $9.92 \mathrm{E}+01$ & $1.44 \mathrm{E}+03$ & $1.77 \mathrm{E}+03$ & $1.05 \mathrm{E}+03$ & $7.33 \mathrm{E}+02$ \\
\hline \multirow{5}{*}{ S\&P 100 } & MED & $2.93 \mathrm{E}-06$ & $\mathbf{1 . 5 6 E}-06$ & $7.34 \mathrm{E}-05$ & $6.20 \mathrm{E}-04$ & $7.90 \mathrm{E}-04$ \\
& VRE & $6.53 \mathrm{E}-01$ & $7.28 \mathrm{E}-02$ & $3.60 \mathrm{E}+00$ & $6.10 \mathrm{E}-01$ & $6.90 \mathrm{E}-01$ \\
& MRE & $1.32 \mathrm{E}-02$ & $1.05 \mathrm{E}-02$ & $9.75 \mathrm{E}-01$ & $2.10 \mathrm{E}-01$ & $2.50 \mathrm{E}-01$ \\
& Runtime(s) & $\mathbf{1 . 2 9 E}+\mathbf{0 2}$ & $1.85 \mathrm{E}+03$ & $2.28 \mathrm{E}+03$ & $1.35 \mathrm{E}+03$ & $9.43 \mathrm{E}+02$ \\
\hline \multirow{5}{*}{ Nikkei } & MED & $1.02 \mathrm{E}-06$ & $\mathbf{8 . 3 3 E - 0 7}$ & $4.03 \mathrm{E}-05$ & $1.50 \mathrm{E}-03$ & $2.90 \mathrm{E}-04$ \\
& VRE & $1.23 \mathrm{E}-01$ & $6.36 \mathrm{E}-02$ & $3.18 \mathrm{E}+00$ & $2.10 \mathrm{E}-01$ & $4.30 \mathrm{E}-01$ \\
& MRE & $1.76 \mathrm{E}-02$ & $1.34 \mathrm{E}-02$ & $8.32 \mathrm{E}-01$ & $9.30 \mathrm{E}-01$ & $1.40 \mathrm{E}-01$ \\
& Runtime(s) & $\mathbf{1 . 3 2 E + 0 3}$ & $1.84 \mathrm{E}+04$ & $2.27 \mathrm{E}+04$ & $1.35 \mathrm{E}+04$ & $9.40 \mathrm{E}+03$ \\
\hline
\end{tabular}

Table 2. The test results of DEaf-MOPS/D algorithm for Test 1.2

\begin{tabular}{lllll}
\hline Data & MED & VRE & MRE & Runtime(s) \\
\hline HangSeng & $7.46 \mathrm{e}-04$ & $4.32 \mathrm{e}+01$ & $4.94 \mathrm{e}+00$ & 2294.69228 \\
DAX 100 & $7.16 \mathrm{e}-04$ & $5.96 \mathrm{e}+01$ & $1.58 \mathrm{e}+00$ & 6614.481647 \\
FTSE 100 & $4.03 \mathrm{e}-04$ & $4.37 \mathrm{e}+01$ & $1.60 \mathrm{e}+00$ & 7192.021137 \\
S\&P 100 & $8.47 \mathrm{e}-04$ & $5.95 \mathrm{e}+01$ & $2.79 \mathrm{e}+00$ & 8224.338064 \\
Nikkei & $2.48 \mathrm{e}-04$ & $3.20 \mathrm{e}+01$ & $8.64 \mathrm{e}-01$ & 37559.665309 \\
\hline
\end{tabular}



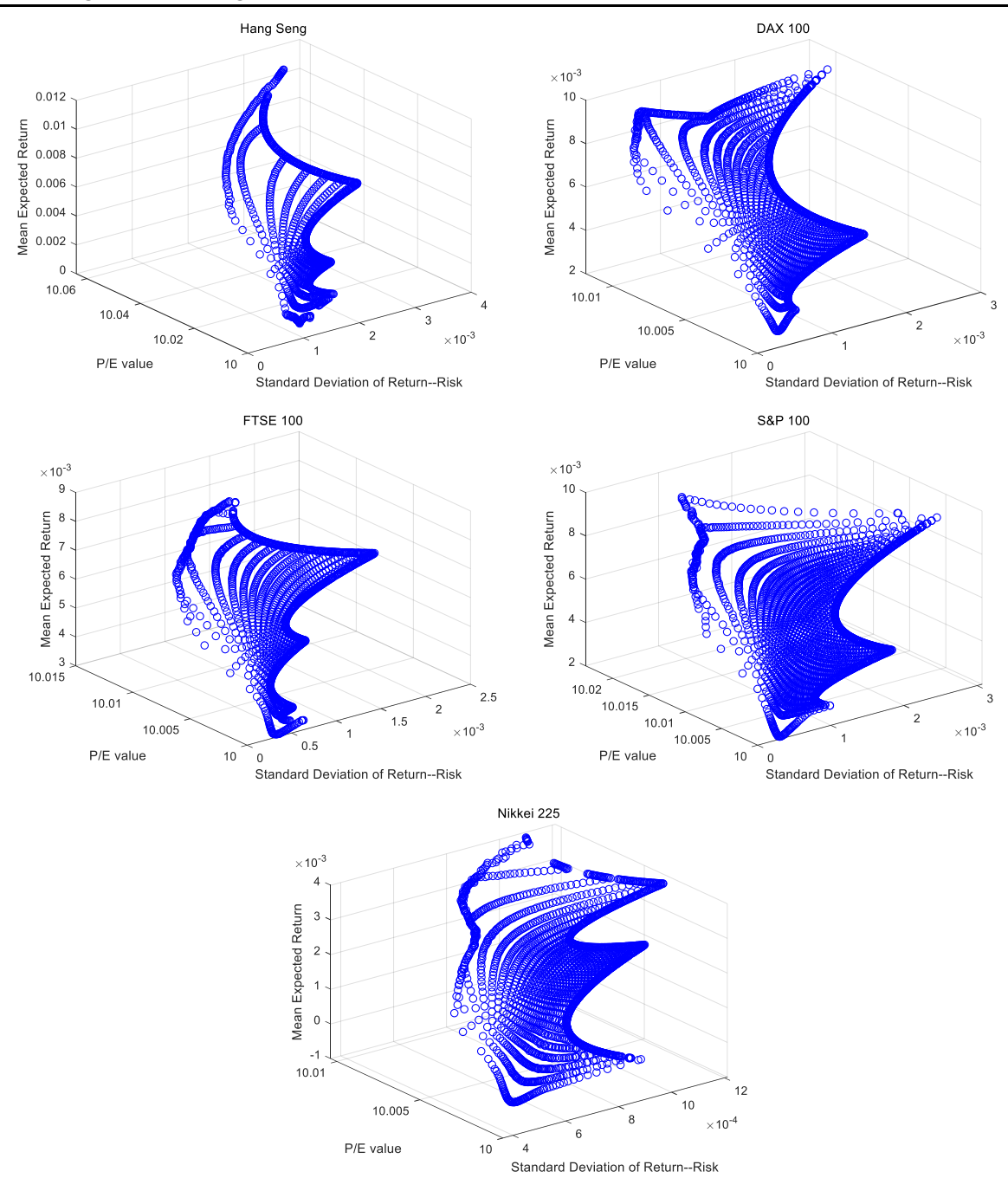

Figure 3. The optimal frontiers of three objectives using DEaf-MOPS/D

\subsection{Constraint tests.}

The cardinality $\mathrm{K}=\min (0.1 \mathrm{D}, 20)$ for the cardinality constraint. The constraint of portfolio weights proportion of each asset: $\varepsilon^{L}=0.01, \varepsilon^{U}=0.5$. For the transaction cost constraint, the fixed transaction cost is equal to one thousandth of one millimeter of mean expected return and the variable transaction 
DEaf-MOPS/D: An Improved Differential Evolution Algorithm for Solving Complex Multi-objective Portfolio Selection Problems Based on Decomposition

cost of $\mathrm{i}^{\text {th }}$ asset is set to $3 \mathrm{x}_{\mathrm{i}} \mathrm{r}_{\mathrm{i}} / 1000$. For the constraint tests, we also investigate two objective and three objective functions, respectively.

(Test 2.1) Two objective functions with cardinality constraint, but without transaction cost constraint. Table 3 summarizes the test results of three algorithms (DEaf-MOPS/D, HS-TLBO and HSDS).

(Test 2.2) Two objective functions with transaction cost constraint. The experimental results are summarized in Table and Figure 4.

(Test 2.3) Three objective functions. In Test 2.3, our algorithm DEaf-MOPS/D is investigated. The surface of optimal frontiers is illustrated in Figure 5.

Table 3. The experimental results of Test 2.1

\begin{tabular}{lllll}
\hline Data & Metric index & DEaf-MOPS/D & HS-TLBO & HSDS \\
\hline \multirow{5}{*}{ HangSeng } & MED & $\mathbf{5 . 2 2 4 5 E - 0 5}$ & $9.3584 \mathrm{E}-05$ & $7.8019 \mathrm{E}-05$ \\
& VRE & $\mathbf{3 . 4 2 8 7 E + 0 0}$ & $4.8186 \mathrm{E}+00$ & $1.6603 \mathrm{E}+00$ \\
& MRE & $\mathbf{2 . 6 2 9 6 E - 0 1}$ & $5.7416 \mathrm{E}-01$ & $6.3577 \mathrm{E}-01$ \\
& Runtime(s) & $\mathbf{1 . 6 4 7 0 E + 0 2}$ & $5.4460 \mathrm{E}+02$ & $6.5352 \mathrm{E}+02$ \\
\hline \multirow{5}{*}{ DAX 100 } & MED & $\mathbf{5 . 7 9 7 9 E - 0 5}$ & $7.6153 \mathrm{E}-05$ & $7.5593 \mathrm{E}-05$ \\
& VRE & $\mathbf{6 . 9 0 0 1 E + 0 0}$ & $7.2802 \mathrm{E}+00$ & $7.0957 \mathrm{E}+00$ \\
& MRE & $\mathbf{2 . 5 7 8 4 E - 0 1}$ & $5.0561 \mathrm{E}-01$ & $5.0335 \mathrm{E}-01$ \\
& Runtime(s) & $\mathbf{7 . 3 6 1 0 E + 0 2}$ & $2.5550 \mathrm{E}+03$ & $3.0660 \mathrm{E}+03$ \\
\hline \multirow{5}{*}{ FTSE 100 } & MED & $\mathbf{2 . 9 4 4 2 E - 0 5}$ & $9.1548 \mathrm{E}-05$ & $7.6611 \mathrm{E}-05$ \\
& VRE & $\mathbf{3 . 3 3 7 4 E + 0 0}$ & $8.5931 \mathrm{E}+00$ & $7.5298 \mathrm{E}+00$ \\
& MRE & $\mathbf{1 . 7 8 4 1 E - 0 1}$ & $6.0975 \mathrm{E}-01$ & $4.9958 \mathrm{E}-01$ \\
& Runtime(s) & $\mathbf{7 . 7 3 5 1 E + 0 2}$ & $2.6327 \mathrm{E}+03$ & $3.1593 \mathrm{E}+03$ \\
\hline \multirow{5}{*}{ S\&P 100 } & MED & $\mathbf{3 . 5 9 3 2 E - 0 5}$ & $1.1515 \mathrm{E}-04$ & $6.7046 \mathrm{E}-05$ \\
& VRE & $\mathbf{3 . 1 9 9 3 E + 0 0}$ & $9.1221 \mathrm{E}+00$ & $5.9638 \mathrm{E}+00$ \\
& MRE & $\mathbf{2 . 5 9 3 1 E - 0 1}$ & $1.1053 \mathrm{E}+00$ & $8.2055 \mathrm{E}-01$ \\
& Runtime(s) & $\mathbf{9 . 2 5 2 1 E + 0 2}$ & $3.4858 \mathrm{E}+03$ & $4.1829 \mathrm{E}+03$ \\
\hline \multirow{5}{*}{ Nikkei } & MED & $\mathbf{3 . 8 0 2 5 E - 0 5}$ & $4.0325 \mathrm{E}-05$ & $5.111 \mathrm{E}-05$ \\
& VRE & $1.0585 \mathrm{E}+01$ & $3.1790 \mathrm{E}+00$ & $4.3668 \mathrm{E}+00$ \\
& MRE & $\mathbf{3 . 0 0 4 7 E - 0 1}$ & $8.3200 \mathrm{E}-01$ & $1.0159 \mathrm{E}+00$ \\
& Runtime(s) & $\mathbf{8 . 3 6 2 0 E + 0 3}$ & $3.8344 \mathrm{E}+04$ & $4.6012 \mathrm{E}+04$ \\
\hline
\end{tabular}


Shouheng Tuo, Hong He

\begin{tabular}{|c|c|c|c|c|}
\hline Data & Metric index & DE-MOPS/D & HS-TLBO & HSDS \\
\hline \multirow{4}{*}{ HangSeng } & MED & 6.62E-05 & $1.21 \mathrm{E}-04$ & $1.14 \mathrm{E}-04$ \\
\hline & VRE & $4.10 \mathrm{E}+00$ & $5.55 \mathrm{E}+00$ & $5.15 \mathrm{E}+00$ \\
\hline & MRE & 3.53E-01 & $8.08 \mathrm{E}-01$ & $7.59 \mathrm{E}-01$ \\
\hline & Runtime(s) & $1.86 \mathrm{E}+02$ & $5.43 \mathrm{E}+02$ & $6.82 \mathrm{E}+02$ \\
\hline \multirow{4}{*}{ DAX 100} & MED & 6.43E-05 & $1.23 \mathrm{E}-04$ & $1.22 \mathrm{E}-04$ \\
\hline & VRE & $7.94 \mathrm{E}+00$ & $1.24 \mathrm{E}+01$ & $1.20 \mathrm{E}+01$ \\
\hline & MRE & 2.66E-01 & $7.25 \mathrm{E}-01$ & 7.22E-01 \\
\hline & Runtime(s) & $8.05 E+02$ & $245 E+03$ & $3.07 \mathrm{E}+03$ \\
\hline \multirow{4}{*}{ FTSE 100} & MED & $3.86 \mathrm{E}-05$ & $1.28 \mathrm{E}-04$ & $1.22 \mathrm{E}-04$ \\
\hline & VRE & $4.64 \mathrm{E}+00$ & $1.29 \mathrm{E}+01$ & $1.24 \mathrm{E}+01$ \\
\hline & MRE & 2.21E-01 & $7.91 \mathrm{E}-01$ & 7.46E-01 \\
\hline & Runtime(s) & $8.25 \mathrm{E}+02$ & $2.97 \mathrm{E}+03$ & $3.32 \mathrm{E}+03$ \\
\hline \multirow{4}{*}{ S\&P 100} & MED & $4.94 \mathrm{E}-05$ & $2.59 \mathrm{E}-04$ & $1.63 \mathrm{E}-04$ \\
\hline & VRE & $4.37 \mathrm{E}+00$ & $1.70 \mathrm{E}+01$ & $1.19 \mathrm{E}+01$ \\
\hline & MRE & 3.40E-01 & $2.61 \mathrm{E}+00$ & $1.66 \mathrm{E}+00$ \\
\hline & Runtime(s) & $1.08 E+03$ & $3.81 \mathrm{E}+03$ & $4.72 E+03$ \\
\hline \multirow{4}{*}{ Nikkei } & MED & $6.62 \mathrm{E}-05$ & $1.22 \mathrm{E}-04$ & $1.21 \mathrm{E}-04$ \\
\hline & VRE & $1.18 E+01$ & $1.82 \mathrm{E}+01$ & $1.76 \mathrm{E}+01$ \\
\hline & MRE & 3.42E-01 & $8.62 \mathrm{E}+00$ & $8.37 \mathrm{E}+01$ \\
\hline & Runtime(s) & $9.34 E+03$ & $3.60 \mathrm{E}+04$ & $4.20 \mathrm{E}+04$ \\
\hline
\end{tabular}

For (Test 2.1), we can find evidently from Table 3 that the DEaf-MOPS/D is the winner on all metrics, and its runtime is about one-third of HS-TLBO, and about a quarter of HSDS. In Table 4, we also can see that the DEaf-MOPS/D is superior to HS-TLBO AND HSDS obviously on all four metrics (MED, VRE, MRE and runtime). Figure 4 shows the optimal frontiers of three algorithms for solving Test 2.2. We can find the optimal fronties of DEaf-MOPS/D from it is more evenly distributed than those of HS-TLBO and HSDS. For (Test 2.3), the surfaces of optimal frontiers of DEaf-MOPS/D are distributed evenly for five datasets.

The test results of DEaf-MOPS/D, that are summarized in Table 5, indicate DEaf-MOPS/D is efficient for solving multi-objective models with cardinality constraint and transaction cost constraint. 
DEaf-MOPS/D: An Improved Differential Evolution Algorithm for Solving Complex Multi-objective Portfolio Selection Problems Based on Decomposition

Table 5. The test results of DEaf-MOPS/D algorithm for Test 2.3

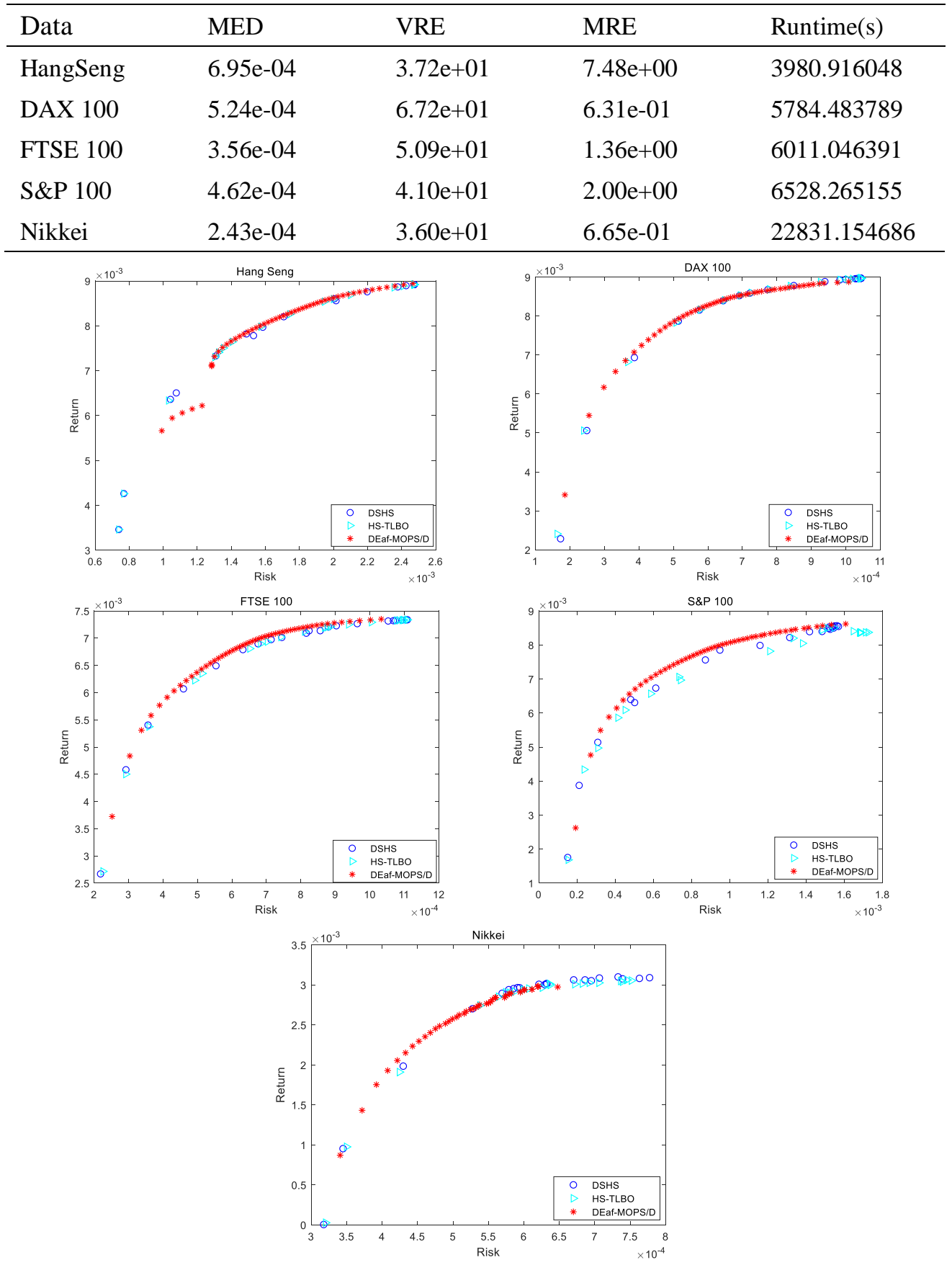

Figure 4. The optimal frontiers of three algorithms for Test 2.2 


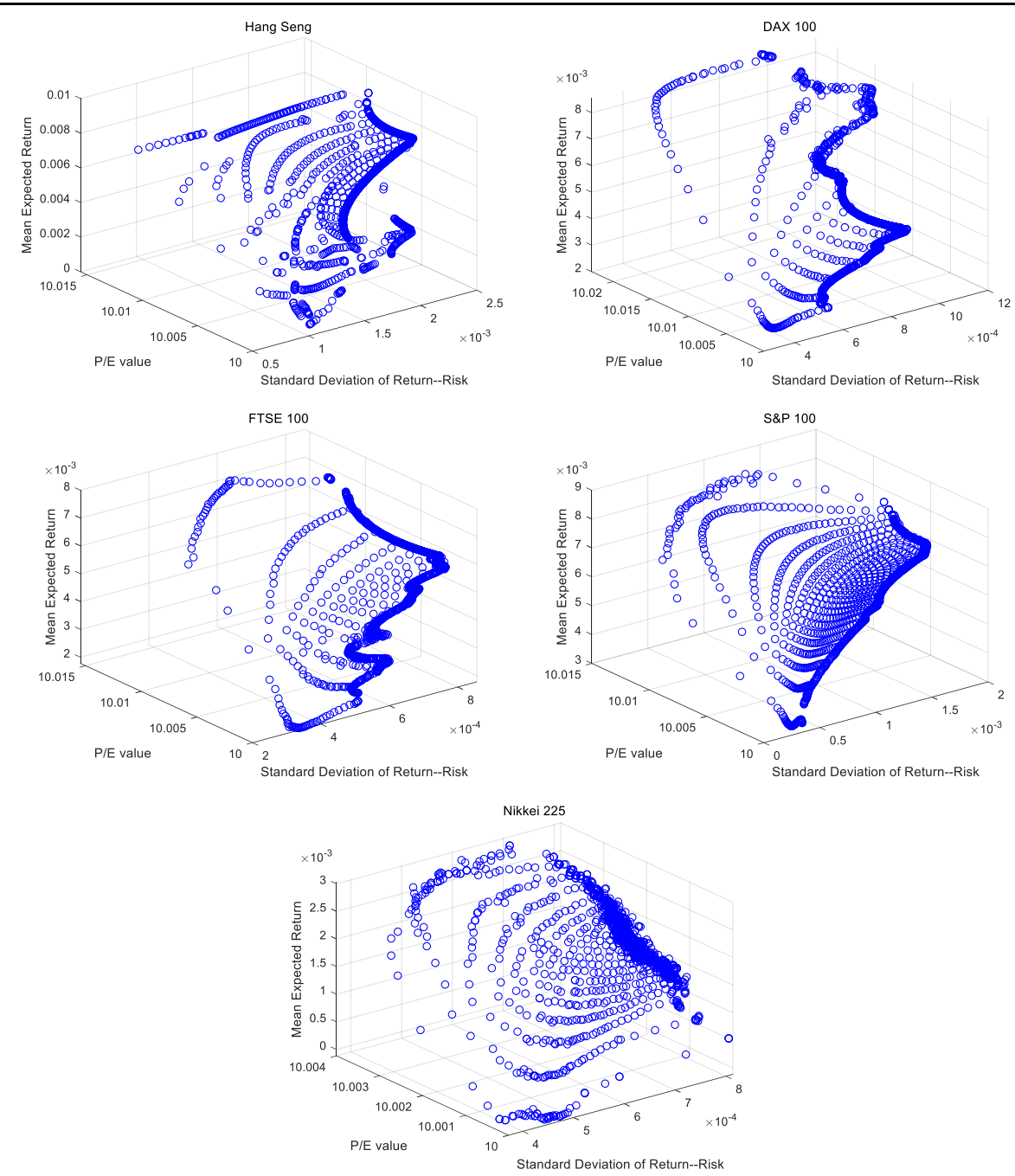

Figure 5. The surface of optimal frontiers of DEaf-MOPS/D for Test 2.3

\section{Conclusion}

In this study, we are intended to solve high-dimensional multi-objective portfolio selection problems. Above all, cardinality constraint mean-variance model with transaction cost constraint and price-earnings ratio is introduced. Then an improved multi-objective differential evolution (DE) algorithm (DEaf-MOPS/D) with fine-tuning strategy are proposed, and decomposition strategy is utilized to solve multi-objective problems. Five simulation datasets are employed to investigate the performance of DEaf-MOPS/D and five differential kinds of tests are performed. The experimental results suggest that, for unconstraint problems, 
DEaf-MOPS/D: An Improved Differential Evolution Algorithm for Solving Complex Multi-objective Portfolio Selection Problems Based on Decomposition the DEaf-MOPS/D is inferior to HS-TLBO on MED, VRE and MRE, but its runtime is much less than that of HS-TLBO. For constraint problems, the DEaf-MOPS/D is superior to comparison algorithms evidently on all four metrics, which demonstrates that the proposed algorithm is promising to solve complex multi-objective portfolio problems.

\section{ACKNOWLEDGEMENTS}

The author would like to thank all the editors, reviewers and referees for their constructive comments. This work was supported by the Ministry of education of Humanities and Social Science project, China under Nos. 19YJCZH148.

\section{REFERENCES}

[1] A.R. Pouya, M. Solimanpur, M.J. Rezaee (2016), Solving Multi-objective Portfolio Optimization Problem Using Invasive Weed Optimization; Swarm Evolut. Comput.28:42-57;

[2] B. Niu, Y. Fan, H. Xiao, B. Xue (2012), Bacterial Foraging Based Approaches to Portfolio Optimization with Liquidity Risk; Neurocomputing 98 (3):90-100;

[3] C.B. Kalayci, O. Ertenlice, H. Akyer, H. Aygoren (2017), An Artificial Bee Colony Algorithm with Feasibility Enforcement and Infeasibility Toleration Procedures for Cardinality Constrained Portfolio Optimization; Expert Syst. Appl. $85: 61-75$;

[4] Chang T J , Meade N, Beasley J E, et al (2000), Heuristics for Cardinality Constrained Portfolio Optimisation[J]. Computers \& Operations Research, 27(13):1271-1302;

[5] E. Zitzler, M. Laumanns, and L. Thiele (2001), SPEA2: Improving the Strength Pareto Evolutionary Algorithm for Multiobjective Optimization[C]// Evolutionary Methods for Design Optimization and Control with Applications to Industrial Problems, K. C. Giannakoglou, D. T. Tsahalis, J. Périaux, K. D. Papailiou, and T. Fogarty, Eds., Athens, Greece. 95-100; 
Shouheng Tuo, Hong He

[6] Ertenlice O, Kalayci C. B. (2018), A Survey of Swarm Intelligence for Portfolio Optimization: Algorithms and Applications. Swarm \& Evolutionary Computation, 39(4): 36-52;

[7] H. Zhu, Y. Wang, K. Wang, Y. Chen (2011), Particle Swarm Optimization (PSO) for the Constrained Portfolio Optimization Problem; Expert Syst. Appl. 38 (8): 10161-10169;

[8] K. Deb, S. Agrawal, A. Pratap, et al. (2002), Meyarivan, A Fast and Elitist Multiobjective Genetic Algorithm: NSGA-II; IEEE Trans. Evol. Comput. 6(2): 182-197;

[9] K. Metaxiotis, K. Liagkouras (2012), Multiobjective Evolutionary Algorithms for Portfolio Management: A Comprehensive Literature Review; Expert Syst. Appl. 39 (14): 11685-11698;

[10] K.Liagkouras, K.Metaxiotis (2018), Multi-period Mean-variance Fuzzy Portfolio Optimization Model with Transaction Costs. Engineering Applications of Artificial Intelligence, 67:260-269;

[11] L. Tan, B. Niu, F. Lin, Q. Duan, L. Li(2013), Modified Bacterial Foraging Optimization for Constrained Portfolio Optimization; Inf. Technol. J. 12 (23):7918-7921;

[12] M. Woodside-Oriakhi, C. Lucas, J.E. Beasley(2011), Heuristic Algorithms for the Cardinality Constrained Efficient Frontier; Eur. J. Oper. Res. 213:538-550;

[13] M.J. Magill, G.M. Constantinides (1976), Portfolio Selection with Transactions Costs; J. Econ. Theor. 13 (2) 245-263;

[14] Markowitz, H. M. (1959), Portfolio Selection: Efficient Diversification of Investments. John Wiley \& Sons;

[15] P. Jorion (1997), Value at Risk: The New Benchmark for Controlling Market Risk. Irwin Professional Pub.;

[16] R.T. Rockafellar, S. Uryasev (2000), Optimization of Conditional Value-at-risk; J. Risk, 2:21-42;

[17] S. Kamali(2014), Portfolio Optimization Using Particle Swarm Optimization and Genetic Algorithm; J. Math. Comput. Sci. 10: 85-90; [18] Strumberger I , Bacanin N , Tuba M (2017), Constrained Portfolio Optimization by Hybridized Bat Algorithm[C]// .International Conference on Intelligent Systems. IEEE, 2017; 
DEaf-MOPS/D: An Improved Differential Evolution Algorithm for Solving Complex Multi-objective Portfolio Selection Problems Based on Decomposition

[19] T.Cura (2009), Particle Swarm Optimization Approach to Portfolio Optimization; Nonlinear Anal.:Real.WorldAppl.10(4): 2396-2406;

[20] T.-J. Chang, N. Meade, J.E. Beasley, Y.M. Sharaiha (2000), Heuristics for Cardinality Constrained Portfolio Optimisation; Comput. Oper. Res.

27:1271-1302;

[21] Tuo S. H. (2016), A Modified Harmony Search Algorithm For Portfolio Optimization Problems[J]. Economic Computation \& Economic Cybernetics Studies \& Research, 50, ASE Publishing, Bucharest;

[22] Tuo S. H., He H. (2018), Solving Complex Cardinality Constrained Mean-variance Portfolio Optimization Problems Using Hybrid HS and TLBO Algorithm. Economic Computation \& Economic Cybernetics Studies \& Research. 52 (3):231-248, ASE Publishing, Bucharest;

[23] Yamazaki, K. H. (1991), Mean-absolute Deviation Portfolio Optimization Model and Its Applications to Tokyo Stock Market. Management Science, 37(5), 519-531;

[24] Zhang Q., Hui L. (2007), MOEA/D: A Multiobjective Evolutionary Algorithm Based on Decomposition[J]. IEEE Transactions on Evolutionary Computation, 11(6):712-731. 